\title{
Serbest Metabelyen Lie Cebirlerinin Direkt Toplamlarının Test Elemanları
}

\author{
Cennet ESKAL ${ }^{1 *}$ (D) Naime EKİCí $\dot{I}^{2}$ \\ ${ }^{1}$ Osmaniye Korkut Ata Üniversitesi, Fen Edebiyat Fakültesi, Matematik Bölümü, Osmaniye, Türkiye \\ ${ }^{2}$ Çukurova Üniversitesi, Fen Edebiyat Fakültesi, Matematik Bölümü, Adana, Türkiye
}

Geliş / Received: 27/11/2018, Kabul / Accepted: 11/07/2019

\section{$\ddot{O} \mathbf{z}$}

$M_{n_{i}}$, rank1 $n_{i}$ olan serbest metabelyen Lie cebiri ve $M, i=1,2, \ldots, m$ için $M_{n_{i}}$ cebirlerinin direkt toplamı olsun. Bu çalışmada $M$ nin bir test elemanına sahip olması için gerek ve yeter koşulun her $i$ için $n_{i}=2$ olduğu ispatlanmıştır. Ayrıca $u=u_{1}+u_{2}+\cdots+u_{m} \in M$ olmak üzere " $u$ nun bir test elemanı olması için gerek ve yeter koşul $i=1,2, \ldots, m$ için $u_{i} \in M_{2}^{\prime}$ ve $\left\{u_{1}, u_{2}, \cdots, u_{m}\right\}$ kümesinin bağımsız olmasıdır" sonucu elde edilmiştir.

Anahtar Kelimeler: Test elemanları, direkt toplam, serbest Lie cebirleri

\section{Test Elements of Direct Sums of Free Metabelian Lie Algebra}

\begin{abstract}
Let $M_{n_{i}}$ be the free metabelian Lie algebra of rank $n_{i}$ and $M$ be the direct sum of $M_{n_{i}}, i=1,2, \ldots, m$. In this paper it is proved that $M$ has a test element if and only if $n_{i}=2$ for each $i$. Additionally it is obtained that an element $u=u_{1}+u_{2}+\cdots+u_{m} \in M$ is a test element if and only if $u_{i} \in M_{2}^{\prime}$ for $i=1,2, \ldots, m$ and $\left\{u_{1}, u_{2}, \cdots, u_{m}\right\}$ is an independent set.
\end{abstract}

Keywords:

\section{Giriş}

$K$ bir cisim ve $F, K$ üzerinde bir serbest Lie cebiri olsun. $F$ nin her $\varphi$ endomorfizmi ve bir $u \in F$ için $\varphi(u)=u$ olduğunda $\varphi$ bir otomorfizm oluyorsa $u$ ya $F$ nin bir test elemanı denir. Serbest gruplar ve serbest cebirlerin test elemanları Dick (1982), Drensky ve Yu (1998), Shpilrain (1995) ve Turner (1996) 1n çalışmalarında önemli bir yer tutmuştur. Serbest Lie cebirleri için de çeşitli test elemanı örnekleri verilmiştir (Esmerligil ve Ekici, 2006; Mikhalev ve Yu, 1997 ve 2000; Mikhalev vd. 2001; Temizyürek ve Ekici, 2006 ve 2007 ). Lie cebirlerinin çarpımları için test elemanlarının varlığ araştırılmıştır. Ekici ve Öğüşlü (2011), bir serbest abelyen Lie cebiri ile bir serbest Lie cebirinin abelyen çarpımının bir test elemanına sahip olduğunu göstermişlerdir. Bununla birlikte serbest abelyen Lie cebirlerinin çözülebilir çarpımının test elemanına sahip olmadığ1 Öğüşlü ve Ekici (2018) de gösterilmiştir. Sonlu rankl1 serbest Lie cebirlerinin sonlu direkt toplamlarının ve serbest çarpımlarının test elemanlarına sahip olması için gerek ve yeter koşullar Eskal ve Ekici (2016) tarafından araştırılmıştır.

Serbest Lie cebirlerinin direkt toplamlarının test elemanlarının varlığı, serbest metabelyen Lie cebirlerinin direkt toplamı için de test elemanlarının var olup olmadığı problemini düşündürmüştür.

Biz bu çalışmamızda sonlu ranklı serbest metabelyen Lie cebirlerinin direkt toplamının test elemanlarının varlığ 1 için gerek ve yeter koşulları inceledik. 


\section{Temel Bilgiler}

$K$ karakteristiği sıfır olan bir cisim olsun. $M_{n_{1}}, M_{n_{2}}, \ldots, M_{n_{m}}, \quad K$ üzerinde rankları sirasiyla $n_{1}, n_{2}, \ldots, n_{m}$ olan serbest metabelyen Lie cebirleri ve $M=\oplus_{i=1}^{m} M_{n_{i}}$ olsun. $M^{\prime}$ ile $M$ nin türetilmiş alt cebirini gösterelim.

Tanım 2.1. $i=1,2, \ldots, m$ için $u_{i} \in M_{n_{i}}$ olsun. $i \neq j$ için $\theta\left(u_{i}\right)=u_{j}$ olacak şekilde $\theta: M_{n_{i}} \rightarrow M_{n_{j}} \quad$ homomorfizmi yoksa $\left\{u_{1}, u_{2}, \ldots, u_{m}\right\}$ kümesi bă̆ımsızdır denir.

Temel sonucumuzun ispatında aşağıdaki teorem önemli bir rol oynamaktadır.

Teorem 2.2. (Esmerligil ve Ekici, 2003) $\quad M$ rank1 2 olan serbest metabelyen Lie cebiri olsun. O zaman $M$ nin yegane test elemanları, $M^{\prime}$ cebirinin elemanlarıdır.

Tanım 2.3. $M_{n}$ rankı $n \geq 2$ olan serbest metabelyen Lie cebiri ve $r \leq n$ olmak üzere $\left\{w_{1}, w_{2}, \ldots, w_{r}\right\}, M_{n}$ nin bir alt kümesi olsun. $M_{n}$ nin her $\varphi$ endomorfizmi için $\varphi\left(w_{i}\right)=w_{i}$, $i=1,2, \ldots, r$ koşulları $\varphi$ nin bir otomorfizm olmasını gerektiriyorsa $\left\{w_{1}, w, \ldots, w_{r}\right\}$ kümesine $M_{n}$ nin bir test kümesi, test kümesinin eleman sayısına da $M_{n}$ nin test rankl denir.

Bu çalışmada yararlandığımız diğer bir sonuç da aşağıdadır (Esmerligil ve Ekici, 2003; Esmerligil vd., 2006).

Teorem 2.4. $M_{n}$ rank1 $n \geq 2$ olan serbest metabelyen Lie cebiri olsun. O zaman $M_{n}$ nin test rank1 $n-1$ dir.

\section{Temel Sonuçlar}

Teorem 3.1. $M=\bigoplus_{i=1}^{m} M_{n_{i}}$ nin bir test elemanına sahip olması için gerek ve yeter koşul her $i=1,2, \ldots, m$ için $n_{i}=2$ olmasidir.

İspat: $u=u_{1}+u_{2}+\cdots+u_{m}, \quad M \operatorname{nin}$ bir test elemanı olsun. Burada $i=1,2, \ldots, m$ için $u_{i} \in M_{n_{i}}$ dir. $u_{1}$ in $M_{n_{1}}$ için bir test elemanı olmadığını kabul edelim. O zaman $\varphi_{1}\left(u_{1}\right)=u_{1}$ olacak şekilde $M_{n_{1}}$ in otomorfizm olmayan bir endomorfizmi vardir.

Şimdi $\varphi: M \rightarrow M, \quad \varphi(u)=\varphi_{1}\left(u_{1}\right)+u_{2}+$ $\cdots+u_{m}$ olarak tanımlanan homomorfizmi düşünelim. Bu durumda

$$
\varphi(u)=\varphi_{1}\left(u_{1}\right)+u_{2}+\cdots+u_{m}=u_{1}+u_{2}+\cdots+u_{m}=u
$$

olduğu görülür. $\varphi_{1}$ in çekirdeği $\varphi$ nin çekirdeği tarafından içerilmekte olup $\varphi_{1}$ otomorfizm olmadığından $\operatorname{Çek} \varphi \neq\{0\}$ dir. Yani $\varphi$ bir otomorfizm değildir. $\mathrm{Bu}$ ise $u$ nun bir test elemanı olması ile çelişir. $\mathrm{O}$ halde $u_{1}$, $M_{n_{1}}$ için bir test elemanıdır. Teorem 2.4 den $M_{n_{1}}$ in test rank1 $n_{1}-1$ olup $u_{1}$ test eleman olduğundan $n_{1}-1=1$ olup buradan $n_{1}=2$ olduğu elde edilir. Benzer şekilde düşünülürse $n_{2}=\cdots=n_{m}=2$ olduğu elde edilir.

Tersine her $i=1,2, \ldots, m$ için $\quad n_{i}=2$ olduğunu kabul edelim. O zaman Teorem 2.2 den $M_{n_{i}}$ cebirleri test elemanına sahiptirler. $i=1,2, \ldots, m$ için $M_{n_{i}}$, rankı 2 olan serbest metabelyen Lie cebirleri ve $M=M_{n_{1}} \oplus M_{n_{2}}$ $\oplus \cdots \oplus M_{n_{m}}$ olsun. $M$ nin bir test elemanına sahip olduğunu göstereceğiz. $i=1,2, \ldots, m$ için $u_{i}, M_{n_{i}}$ de bir test elemanı olmak üzere

$$
u=u_{1}+u_{2}+\cdots+u_{m}
$$

elemanının $M$ de bir test elemanı olduğunu gösterelim. En az bir $i$ için $u_{i} \neq 0$ olsun. $\varphi: M \rightarrow M, \quad \varphi(u)=u \quad$ olacak şekilde bir homomorfizm olsun. $\varphi$ nin $M_{n_{i}}$ ye kısıtlanışını da $\varphi_{i}$ ile gösterelim. Yani $\varphi_{i}=$ $\left.\varphi\right|_{M_{n_{i}}}$ olsun. $\varphi(u)=u$ eşitliğinden

$$
\varphi(u)=\sum_{i=1}^{m} \varphi_{i}\left(u_{i}\right)=\sum_{i=1}^{m} u_{i}
$$


ve

$$
\sum_{i=1}^{m}\left(\varphi_{i}\left(u_{i}\right)-u_{i}\right)=0, \quad i=1,2, \ldots, m
$$

elde edilir. $\quad M=\oplus_{i=1}^{m} M_{n_{i}}$ olduğundan

$$
\varphi_{i}\left(u_{i}\right)=u_{i}, \quad i=1,2, \ldots, m
$$

olduğu görülür. $u_{i} \in M_{n_{i}}$ test elemanı olduğundan $\quad i=1,2, \ldots, m$ için $\varphi_{i}$ homomorfizmi $\varphi: M_{n_{i}} \rightarrow M_{n_{i}}$ şeklinde bir otomorfizmdir. $i \neq j$ için $\varphi: M_{n_{i}} \rightarrow M_{n_{j}}$ olamaz. Çünkü öyle olsaydı, $\varphi_{i}\left(u_{i}\right)=u_{j}$ olup $\quad \varphi(u)=u \quad$ eşitliğinden $\quad u_{i}=0$ çelişkisi elde edilir.

Şimdi $\varphi \operatorname{nin} M$ nin bir otomorfizmi olduğunu gösterelim. $v=v_{1}+v_{2}+\cdots+v_{m} \in \operatorname{Ç} e k \varphi$ olsun. O zaman

$$
\varphi(v)=\sum_{i=1}^{m} \varphi_{i}\left(v_{i}\right)=0
$$

dir. $\varphi_{i}$ lerin hepsi otomorfizm olduğundan $i=$ $1,2, \ldots, m$ için $v_{i}=0$ ve dolayısıyla $v=0$ bulunur. $\mathrm{O}$ halde $\varphi$ injektiftir.

Her $\quad w=w_{1}+w_{2}+\cdots+w_{m} \in M$ için $w_{i}=\varphi_{i}\left(v_{i}\right) \quad$ olacak şekilde $\quad v_{i} \in M_{n_{i}}$ var olup

$$
w=\varphi\left(v_{1}+v_{2}+\cdots+v_{m}\right)
$$

dir. Yani $\varphi$ örtendir. $\mathrm{O}$ halde $u, M$ nin bir test elemanıdır.

Sonuç 3.2. $i=1,2, \ldots, m$ için $u_{i} \in M_{n_{i}}$ olmak üzere $u=u_{1}+u_{2}+\cdots+u_{m} \in M$ elemanının bir test elemanı olması için gerek ve yeter koşul

a) her $i=1,2, \ldots, m$ için $u_{i} \in M_{2}^{\prime}$

b) $\left\{u_{1}, u_{2}, \cdots, u_{m}\right\}$ kümesinin bağımsız

olmasidir.

İspat. $i=1,2, \ldots, m$ için $u_{i} \in M_{n_{i}}$ olmak üzere $u=u_{1}+u_{2}+\cdots+u_{m}$ elemanı $M$ için bir test elemanı olsun. O zaman Teorem 3.1 den $n_{i}=2$ olup Teorem 2.2 den $u_{i} \in M_{n_{i}}$ birer test elemanıdır ve $u_{i} \in M_{n_{i}}^{\prime}=M_{2}^{\prime}$ dir.

$\left\{u_{1}, u_{2}, \cdots, u_{m}\right\}$ kümesinin bağımlı olduğunu varsayalım. O zaman bazı $i \neq j$ için $\theta\left(u_{i}\right)=u_{j} \quad$ olacak şekilde $\quad \theta: M_{n_{i}} \rightarrow M_{n_{j}}$ homomorfizmi vardır. $\varphi: M \rightarrow M$ homomorfizmini sabit bir $i$ için

$$
\left.\varphi\right|_{M_{n_{i}}}\left(v_{i}\right)=\varphi_{i}\left(v_{i}\right)=\theta\left(v_{i}\right), \quad v_{i} \in M_{n_{i}}
$$

olarak tanımlayalım. O zaman

$$
\begin{gathered}
\varphi(u)=u_{1}+u_{2}+\cdots+u_{j-1}+\theta\left(u_{i}\right)+u_{j+1} \\
+\cdots+u_{m}=u
\end{gathered}
$$

olup $M_{n_{i}} \subset$ Çek $\varphi$ dir. Yani $\varphi(u)=u$ olduğu halde $\varphi$ bir otomorfizm değildir. Bu da $u$ nun test elemanı olması ile çelişir.

Şimdi tersine her $i=1,2, \ldots, m$ için $u_{i} \in M_{2}^{\prime}$ ve $\left\{u_{1}, u_{2}, \cdots, u_{m}\right\}$ kümesi bağımsız olsun. Teorem 3.1 den

$$
\begin{aligned}
u=u_{1}+u_{2}+\cdots+u_{m} \in M & \\
& =M_{1} \oplus \mathrm{M}_{2} \oplus \cdots \oplus \mathrm{M}_{\mathrm{m}}
\end{aligned}
$$

bir test elemanıdır. Burada $M_{i}$ ler rank1 2 olan serbest metabelyen Lie cebirleridir.

Örnek 3.3. $M_{i},\left\{x_{i}, y_{i}\right\}$ tarafindan üretilen serbest metabelyen Lie cebiri Lie cebiri ve $M=M_{1} \oplus \mathrm{M}_{2} \oplus \cdots \oplus \mathrm{M}_{\mathrm{m}} \quad$ olsun. $\quad i=$ $1,2, \ldots, m$ için $u_{i}=\left[x_{i}, y_{i}\right], M_{i}$ nin test elemanı olup

$$
u=\left[x_{1}, y_{1}\right]+\left[x_{2}, y_{2}\right]+\cdots+\left[x_{m}, y_{m}\right],
$$

$M$ nin bir test elemanıdır.

\section{Kaynaklar}

Dick, W. 1982. "A commutator test for two elements to generate the free algebra of rank two", Bull. London Math. Soc., 14, 48-51.

Drensky, V., Yu, J. T. 1998. "Orbits in free algebras of rank two", Comn. Alg., 26, 18951906. 
Ekici,N., Öğüşlü, N. Ş. 2011. "Test rank of an abelian product of a free Lie algebra and a free abelian Lie algebra", Proc. Indian Acad. Sci.(Math. Sci.), 121,(3), 291-300.

Eskal, C., Ekici, N. 2016. "Test elements of direct sum and free products of free Lie algebras", Proc. Indian Acad. Sci.(Math. Sci.), 126(1), 43-48.

Esmerligil, Z., Ekici, N. 2003. Test sets and test rank of free metabelian Lie algebra", Comn. Alg., 31(11), 5581-5589.

Esmerligil, Z., Kahyalar, D., Ekici, N. 2006. "Test rank of $F / R^{\prime}$ Lie algebras", Int. $J$. Alg. Comp., 16(4), 817-825.

Esmerligil, Z., Ekici, N. 2006. "Rank1 2 olan $F / R^{\prime}$ Lie cebirleri için komutatör testi", XIX. Ulusal Matematik Sempozyumu, Konferans Kitab1, Kütahya, 685-690.

Mikhalev, A. A., Yu J. T. 1997. "Test elements and retracts of free Lie algebras", Commun. Alg., 25, 3283-3289.

Mikhalev, A. A., Yu J. T. 2000. "Primitive, almost primitive, test and $\Delta$-primitive elements of free algebras with the NielsenSchrier property", J. Alg., 228, 603-623.

Mikhalev, A. A., Umirbaev, U. U., Yu J. T. 2001. "Generic, almost primitive and test elements of free Lie algebras", Proc. Amer. Math. Soc., 130(5), 1303-1310.

Ögüşlü, N. Ş., Ekici, N. 2018. "The test rank of a solvable a product of free abelian Lie

algebras", J. Alg. Appl.,

doi. 10.1142/50219492819500257.
Shpilrain, V. 1995. "Test elements for endomorphisms of free groups and algebras", Israel J. Math., 92, 307-316.

Temizyürek, A., Ekici, N. 2006. " $F /\left[R^{\prime}, F\right]$ Lie cebirleri için komutatör testi", XIX. Ulusal Matematik Sempozyumu, Konferans Kitabı, Kütahya, 109-115.

Temizyürek, A., Ekici, N. 2007. "A particular test element of a free solvable Lie algebra of rank two", Rocky Mountain J. Math., 37(4), 1415-1426.

Turner, E. C. 1996. "Test words for automorphisms of free groups", Bull. London Math. Soc., 28, 255-263. 